\title{
A Proposed New Method for Damping Relaxation Oscillations in Laser Diodes
}

\author{
J. KATZ, MEMBER, IEEE, S. MARGALIT, AND A. YARIV, FELLOW, IEEE
}

\begin{abstract}
A new concept of damping relaxation oscillations in injection laser diodes is described. This method involves the operation of the laser as a part of a bipolar transistor, and the damping is accomplished by reducing the carrier lifetime in the laser active region only at frequencies near resonance. The advantage of the proposed method is that the damping mechanism does not affect the laser operation at any other frequency range.
\end{abstract}

M

ANY applications of injection laser diodes call for their modulation at high frequencies. Except the phenomenon of self-pulsation, the two main parameters of the laser frequency transfer function are the resonance frequency which roughly sets the upper limit on the useful range where the laser can be modulated, and the magnitude of the resonance peak, which manifests itself in the phenomenon of relaxation oscillations in the pulse response of the laser [1]. From the laser rate equations [1] it is clearly seen that the resonance peak is reduced when the effective lifetime of the carriers in the active region is reduced. This reduction can be achieved either by connecting an impedance in parallel with the laser diode [2], or by utilizing the mechanism of carrier diffusion out of the active region [3-5].

This paper proposes an alternative method of damping the relaxation oscillations. The problem with internal diffusion damping mechanism [3-5] and with connecting untuned impedances in parallel with the laser is that they consume a significant fraction of the laser current even at frequencies far from resonance, where damping is not needed. This problem can be somewhat circumvented by connecting a tuned impedance in parallel with the laser [2], but these external circuits become ineffective when the resonance frequency of the laser changes, for example, due to aging or due to change in the laser bias level. The proposed method solves the above problems. In this new method; the laser possesses different effective carrier lifetimes: one for $\mathrm{dc}$ and low-frequency ac operation, and one for operation at frequencies near the resonance. The laser operates as part of a bipolar transistor with a shorting capacitance (Fig. 1(a)). The basic device structure is shown in Fig. 1(b). Note that the laser active region is part of the transistor emitter.

Manuscript received August 9, 1982.

J. Katz is with the Jet Propulsion Laboratory, California Institute of Technology, Pasadena, CA 91109. S. Margalit and A. Yariv are with the Dept. of Electrical Engineering, California Institute of Technology, Pasadena, CA 91125.

The research described in this paper was carried out by the Jet Propulsion Laboratory, California Institute of Technology, under contract with the National Aeronautics and Space Administration. Portions of this research were also sponsored by the Office of Naval Research and the National Science Foundation.

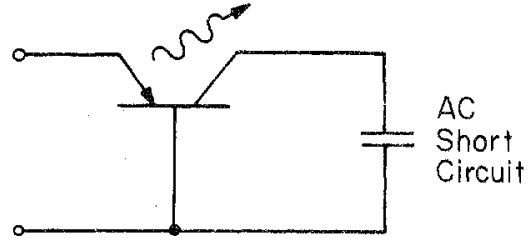

(a)

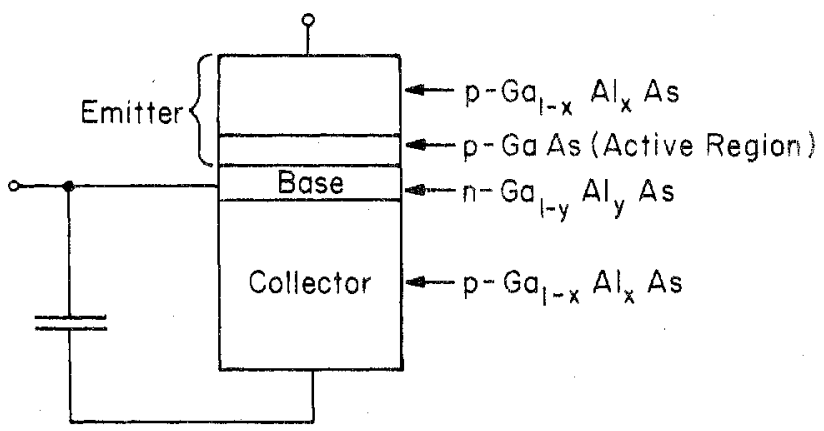

(b)

Fig, 1. Proposed damping method: (a) Schematic drawing of the de vice, (b) Basic structure of the device $(y<x \cong 0.4)$. The width of the active region is $d$, and the width of the base is $w$.

The principle of operation of the proposed cevice cun be understood from Fig. 2, which depicts the hole concentration profiles in the active region and in the base. Parameters with a superscript 0 are dc values, and parameters with a superscript 1 denote small signal ac components. At dc (Fig. 2(a)) there is only a small reduction in the effective carrier lifetime due to recombination of carriers leaking from the active region. into the base. Since the quasi-fermi levels are clamped for frequencies below the resonance, no ac component of the carriers can develop at these frequencies (Fig. 2(b)), and thus the effective carrier lifetime is unchanged. However, for nearresonance frequencies, the populations of the photons and the carriers decouple, and thus an appreciable ac comporent of the carrier density is established in the active region (Fig. $2(c)$ ). The large diffusion current due to the established cartier den" sity gradient in the base (note that the collector-base function is ac-shorted, and thus the excess carrier concentration there is clamped to zero) causes an enhanced leakage current out of the active region, resulting in a reduction of the effective carrier liftime there. The above analysis is done quantitatively in the following paragrpahs.

At dc, the collector terminal (denoted by $\mathrm{C}$ in Fig. 2) is floating, and thus both the emitter and the collector juncm tions are forward biased. The excess hole concentration at the active region (width $d$ ) and the base (width $w$ ) is shown 


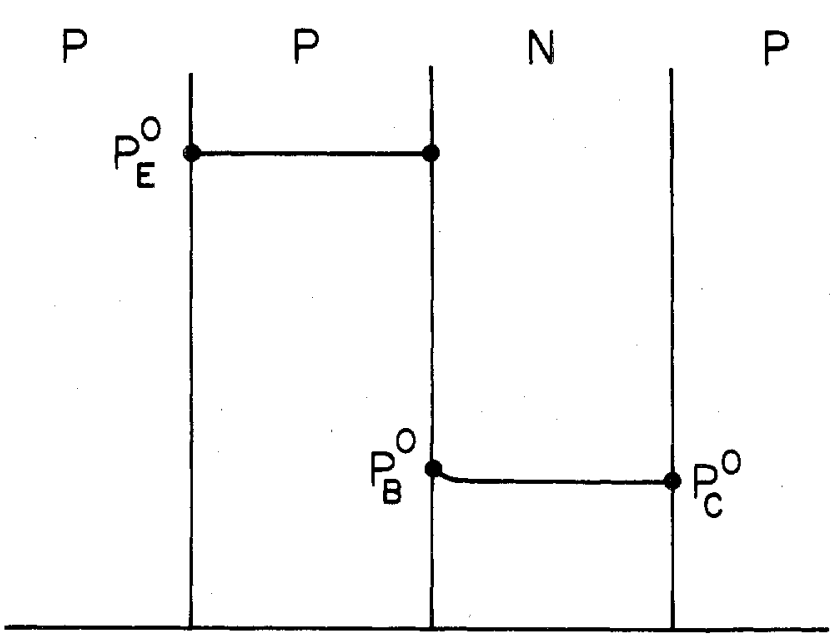

(a)

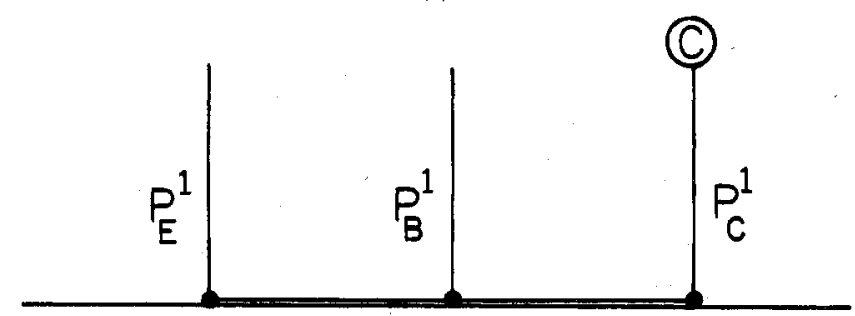

(b)

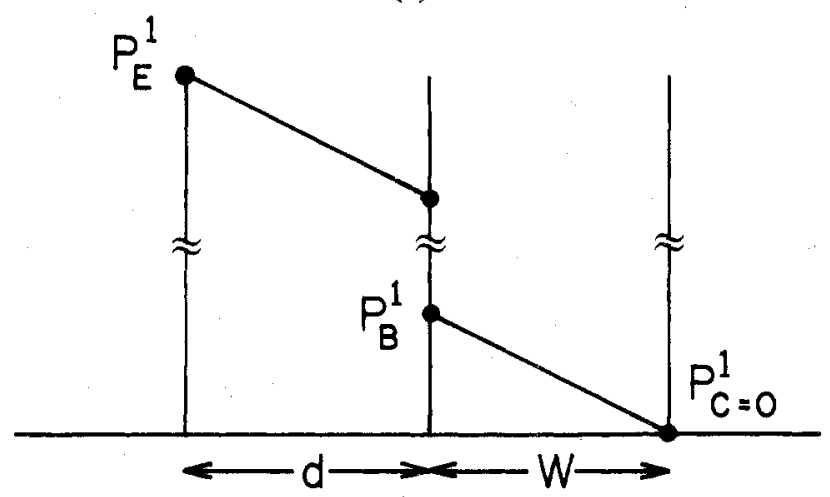

(c)

Fig. 2. Excess carriers concentration profile at the active region and at the base of the transistor: (a) dc, (b) ac, frequencies far from resonance, (c) ac, frequencies near resonance.

in Fig. 2(a). This paper will use the simple charge-control calculations throughout. (Also, since the transistor operates in the common base mode, its cutoff frequency is usually much higher than the laser resonance frequency, so that low frequency conditions can be assumed.) As a first approximation the active region excess carrier concentration $N_{E}{ }^{0}=$ $P_{E}{ }^{0}$ is constant, and the concentration across the base is also approximately constant (since voltage variations are of the order of only $K T / q)$. We assume that $P_{c}{ }^{0} \cong P_{B}{ }^{0}=\delta P_{E}{ }^{0}$, where $\delta$ is a factor determined by the Boltzmann statistics from the bandgap difference $\Delta E$ between the active region and the base (under the applicable high injection conditions, $\delta=\exp [-\Delta E / 2 K T]$. As seen below, in order to obtain values of $\delta$ for operations of practical devices, the fraction $x$ of aluminum in the base should be 0.1 to 0.15 . In this range of values of $x$ the optical operation of the device is similar to that of a large optical cavity laser). As far as the laser operation is considered, the amount of current wasted in the dc operation is lost only by recombination current in the base

$$
J_{\text {leakage }} \mathrm{dc}=q \frac{\delta P_{E}^{0}}{\tau_{s}} w
$$

By including the (negative) contributions of this leakage current in the rate equation for the carriers [1] we see that the effective dc lifetime is reduced from $\tau_{s}$ to $\tau_{s} /(1+\delta w / d)$, which is a negligible change if $\delta \ll 1$ and $w \leqslant d$. In ac operation $\tau_{\text {eff }}$ does not change and is equal to $\tau_{s}$ as long as we are below the resonance region. Since the carriers concentration in the active region is effectively clamped in these frequencies, the ac component of the carriers concentration is virtually zero (Fig. 2(b)). Thus we come to the important conclusion that in this useful frequency region the modulation efficiency (or, equivalently, the differential quantum efficiency) are not affected. Only when we approach resonance, an appreciable ac component of the carriers concentration can be maintained in the active region. The carriers concentration at the base side of the emitter-base junction still follows on according to $P_{B}{ }^{1}=\delta \cdot P_{E}{ }^{1}$, but $P_{C}{ }^{1}=0$ because the collector junction $c$ is ac shorted (Fig. 2(b)). This causes a much larger ac current density from the active region, approximately given by

$$
J_{\text {leakage }}{ }^{\mathrm{Res}}=\frac{q D \delta \cdot P_{E}{ }^{0}}{w}
$$

( $D$ is the carriers diffusion constant), which, when applied in the rate equation for the carriers is seen to reduce the effective carriers ac lifetime for $\tau_{s}$ to

$$
\tau_{\text {eff }}^{\text {Res }}=\frac{\tau_{s}}{1+\frac{\tau_{s} D \delta}{w d} \rho}
$$

For example, for $D=8 \mathrm{~cm}^{-1}, \delta=0.1 \mathrm{w}=0.1 \mu \mathrm{m}, d=0.2$ $\mu \mathrm{m}, \tau_{s}=3 \mathrm{nS}$, and $\rho=1$ (see below) $\tau_{\mathrm{eff}}$ Res is only about $0.23 \mathrm{nS}$. Of course, one has also to consider the effects of the device parasitic elements. When one takes into account the finite resistivities of the external components and of the internal layers of the device, $\rho$ is reduced from 1 to a value given by

$$
\frac{1}{\rho}=a \frac{q D \delta P_{E}^{0}}{w} \frac{q}{2 K T}\left(R_{B}+R_{C}+r_{b b^{\prime}}\right)-1
$$

where $R_{B}$ and $R_{C}$ are the resistances between ground and the collector and base terminals, respectively, $r_{b b}{ }^{\prime}$ is the base spread resistance, and $a$ is the junction cross section area in the direction of the current flow. As an example, for $P_{E}{ }^{0}=1.2 \cdot 10^{18} \mathrm{~cm}^{-3}, R_{B}+R_{C}=4 \Omega, r_{b b}=1 \Omega$ (this low value is attainable with the narrow base widths resulting from the stripe geometry of semiconductor lasers), and $a=$ $2 \times 250 \mu^{2}$, the effective carrier lifetime is reduced from $3 \mathrm{nS}$ to slightly above $1 \mathrm{nS}$. It should be noted that the base- 
collector junction capacitance does not degrade the device operation since it is connected in parallel with the external capacitor. Monolithic implementations of the device are also possible, in which case all the capacitance needed for the acshort is provided by the intrinsic junction capacitance.

In conclusion, we have described a new concept of damping relaxation oscillations in laser diodes by electronic means. This method, based on a combined operation of the laser as a part of a bipolar transistor, has the advantage that the damping mechanism is operative only near resonance. Thus the laser operation is not affected at other frequencies. Significant reduction of the resonant peak is expected to be achieved with realizable components and contact resistances.

\section{REFERENCES}

[1] H. Kressel and J. K. Butler, Semiconductor Lasers and Heterojunction Leds, chapt. 17, New York: Academic Press, 1977.

[2] Y. Suematsu and T. Hong, "Suppression of relaxation oscillation in light output of injection lasers by electrical resonance circuit," IEEE J. Quant. Electron. QE-13, pp. 756-762, 1977.

[3] K. Furuya, Y. Suematsu, and T. Hong, "Reduction of resonancelike peak in direct modulation due to carrier diffusion in injection laser," App. Optics, vol. 17, pp. 1949-1952, 1978

[4] N. Chinone, K. Aiki, M. Nakamura, and R. Ito, "Effects of Iateral mode and carrier density profile on dynamic behaviors of semiconductor lasers," IEEE J. Quant. Electron. QE-14, pp. 625$631,1978$.

[5] D. Wilt, K. Y. Lau, and A. Yariv, "The effect of lateral carrier diffusion on the modulation response of a semiconductor laser, " $J$. Appl, Phys., vol. 52, pp. 4970-4974, 1981.

\title{
100 GHz Binary Counter Based On DC SQUID's
}

\author{
C. A. HAMILTON, MEMBER, IEEE, AND FRANCES L. LLOYD
}

\begin{abstract}
A binary counter using bistable dc SQUID's as flip flop circuits is demonstrated. All of the functions: LOAD, COUNT, STORE, READ, and CLEAR can be performed. The use of single flux quantum logic results in high sensitivity $\left(10^{-18} \mathrm{~J}\right.$ input pulse energy), high speed $\left(100 \mathrm{GHz}\right.$ count rate) and low power $\left(10^{-7} \mathrm{~W}\right.$ at $100 \mathrm{GHz}$ count rate).
\end{abstract}

\section{INTRODUCTION}

$\mathbf{T}$ HIS paper describes the practical realization of a SQUID (Superconducting QUantum Interference Device) based binary counter circuit first proposed by Hurrell and Silver in $1978[1,2]$. This counter uses 2 -junction SQUID's which are biased into a bistable operating region and act as flip flops (FF). The single flux quantum transitions between the two stable states produce carry pulses so that a series of these FF SQUID's can operate as a binary ripple counter. Such a counter is potentially capable of counting at frequencies of $100 \mathrm{GHz}$ or more. Other interesting characteristics of the device are its ability to count input pulses with energies calculated to be as small as $10^{-18} \mathrm{~J}$ with power consumption of only $10^{-7} \mathrm{~W}$ at a $100 \mathrm{GHz}$ count rate.

A circuit diagram for two stages of the counter is shown in Fig. 1. The FLIP FLOP SQUID's consist of two Josephson junctions $\mathrm{J} 1$ and $\mathrm{J} 2$ in a superconducting loop of inductance $L_{f f}$. Bistable operation of the FF SQUID's is achieved by simultaneously applying a control current $I_{c}=\Phi_{o} / 2 M_{c}$ and a gate current $I_{g} \cong I_{01}$. Here the flux quantum $\Phi_{0}=2.07$ $\mathrm{pH} \mathrm{mA}, M_{c}$ is the mutual inductance between the control line and the FF SQUID and $I_{01}$ is the critical current of junc-

Manuscript received August 23, 1982; revised Sept. 14, 1982.

The authors are with the Electromagnetic Technology Division, National Bureau of Standards, Boulder, CO 80303.

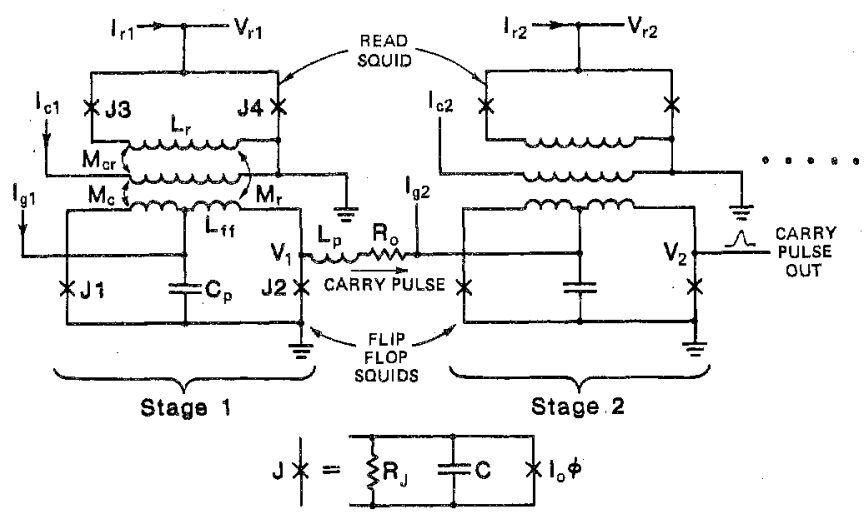

Fig. 1. The circuit for a set of FLIP FLOP and READ SQUID's in a counter configuration. The circuit values used in simulations are $L_{f f}=4.0 \mathrm{pH}, L_{r}=8.6, L_{p}=2.1, M_{c}=M_{\gamma}=1.0, M_{c r}=0.5, I_{01}=$ $I_{02}=0.4 \mathrm{~mA}, I_{03}=I_{04}=0.1, R_{J 1}=0.7 \mathrm{ohms}, R_{J 2}=1.5, R_{0}=$ $0.33, C_{1}=C_{2}=1.4 \mathrm{pF}, C_{3}=C_{4}=0.3, C_{p}=3.2, I_{g} \cong 0.42, I_{c} \cong$ $1.03 \mathrm{~mA}$.

tion $\mathrm{J} 1$ and $\mathrm{J} 2$. The gate current, $I_{g}$, splits and flows downward through $\mathrm{J} 1$ and $\mathrm{J} 2$ to ground. The control current $I_{c}$ induces a clockwise circulating current of approximately $I_{0} / 2$ which adds to the current through $\mathrm{J} 2$ and subtracts from the current through $J 1$. Thus $\mathrm{J} 2$ is biased near its critical current and $\mathrm{J} 1$ is biased near zero. The application of a

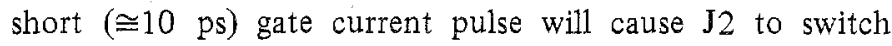
momentarily into the voltage state. The resulting voltage pulse across $L_{f f}$ reverses the direction of the circulating current so the $\mathrm{J} 1$ is biased near its critical current and $\mathrm{J} 2$ is biased near zero. The next gate current pulse results in a voltage pulse across $\mathrm{J} 1$ and a return to the original clockwise circulating current. The requirement of flux quantization 